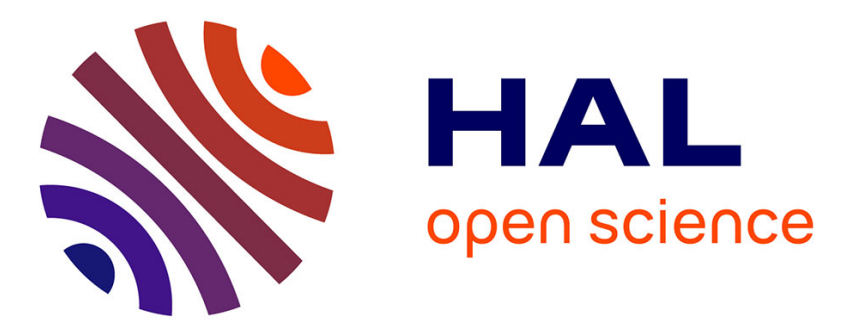

\title{
Two-step polarization reversal in biased ferroelectrics
}

John Daniels, Clayton Cozzan, Supphatuch Ukritnukun, Goknur Tutuncu, Jérome Andrieux, Julia Glaum, Chris Dosch, Wook Jo, Jacob L. Jones

\section{To cite this version:}

John Daniels, Clayton Cozzan, Supphatuch Ukritnukun, Goknur Tutuncu, Jérome Andrieux, et al.. Two-step polarization reversal in biased ferroelectrics. Journal of Applied Physics, 2014, 115 (22), pp.224104. 10.1063/1.4881835 . hal-02326277

\section{HAL Id: hal-02326277 \\ https://hal.science/hal-02326277}

Submitted on 8 Jun 2021

HAL is a multi-disciplinary open access archive for the deposit and dissemination of scientific research documents, whether they are published or not. The documents may come from teaching and research institutions in France or abroad, or from public or private research centers.
L'archive ouverte pluridisciplinaire HAL, est destinée au dépôt et à la diffusion de documents scientifiques de niveau recherche, publiés ou non, émanant des établissements d'enseignement et de recherche français ou étrangers, des laboratoires publics ou privés. 


\section{Two-Step Polarization Reversal in Biased Ferroelectrics}

John E. Daniels, ${ }^{1, a)}$ Clayton Cozzan, ${ }^{1,2}$ Supphatuch Ukritnukun, ${ }^{1}$ Goknur Tutuncu, ${ }^{2}$

Jerome Andrieux, ${ }^{3,4}$ Julia Glaum, ${ }^{1}$ Chris Dosch, ${ }^{2}$ Wook Jo, ${ }^{5}$ and Jacob L. Jones ${ }^{2,6}$

${ }^{1}$ School of Materials Science and Engineering, University of New South Wales, Sydney 2052, Australia

${ }^{2}$ Department of Materials Science and Engineering, University of Florida, Gainesville, FL, 32611, USA

${ }^{3}$ Université Claude Bernard Lyon 1, LMI-UMR CNRS n5615, 69622 Villeurbanne Cedex, France

${ }^{4}$ European Synchrotron Radiation Facility, Grenoble 38000, France

${ }^{5}$ School of Mechanical \& Advanced Materials Engineering, UNIST, Ulsan, Korea

${ }^{6}$ Department of Materials Science and Engineering, North Carolina State University, Raleigh, NC, USA

Polarization reversal in polycrystalline ferroelectrics is shown to occur via two distinct and sequential domain reorientation steps. This reorientation sequence, which cannot be readily discriminated in the overall sample polarization, is made apparent using timeresolved high-energy x-ray diffraction. Upon application of electric fields opposite to the initial poling direction, two unique and significantly different time constants are observed. The first (faster time constant) is shown to be derived by the release of a residual stress due to initial electrical biasing and the second (slower time constant) due to the redevelopment of residual stress during further domain wall motion. A modified domain reorientation model is given that accurately describes the domain volume fraction evolution during the reversal process.

\footnotetext{
a) Correspondence should be addressed to John E. Daniels. Electronic mail: j.daniels@unsw.edu.au
} 


\section{$\underline{\text { Introduction }}$}

Polarization reversal is a central functionality of ferroelectrics wherein the spontaneous polarization is reversed through the application of electric fields. As many ferroelectric materials are also ferroelastic, polarization reversal is often also coupled to ferroelasticity. Thus, while polarization reversal may occur in a single step through the motion of $180^{\circ}$ ferroelectric domain walls, experimental evidence demonstrates that sequential steps involving non- $180^{\circ}$ domain orientation states also occurs. ${ }^{1-3}$ Figure 1 illustrates such a multi-step polarization reversal process in a tetragonal system where domains may exist with approximately $90^{\circ}$ orientation relationships.

Polarization reversal in ferroelectrics is often described by the nucleation and growth of reversed domains. A model for this behavior, introduced by Kolmogorov and Avrami and later extended by Ishibashi (KAI) ${ }^{4-6}$ defines polarization reversal with respect to the volume fraction of reversed domains. The behavior of the one-dimensional case (i.e. the domain boundary moves in one direction after a plate-like nuclei forms) is predicted in the KAI model to be an equation of the form,

$$
\eta(t)=1-e^{-t / \tau}
$$

where $\eta(t)$ is the volume fraction of switched domains at time $t$, and $\tau$ is a time constant dependent upon the electric field magnitude. Fatuzzo and Merz empirically determined that the time constant, $\tau$, in this model is best described by an equation of the form,

$$
\tau(E)=\tau_{0} e^{\left(E_{0} / E\right)^{n}}
$$

where $\tau_{0}$ is the characteristic time, $E$ is the applied field, $E_{0}$ is the activation field, and $n$ is a constant with values typically near unity. ${ }^{7}$ It is often assumed that the net polarization is proportional to the volume fraction of switched domains, and thus the KAI model is used to directly interpret polarization measurements. In these cases, the KAI model has been shown to describe polarization 
reversal in certain epitaxial thin films ${ }^{8,9}$ but does not well describe the behavior of samples with larger characteristic features such as polycrystalline materials where emergent phenomena can occur at longer length and time scales. ${ }^{10-12}$ In particular, the symmetric shape of the KAI model does not fit typical polarization reversal curves because these curves exhibit nonsymmetric and extended quasi-linear tails. ${ }^{13-15}$ These aspects reflect a stretched time-dependent behavior and are thought to be due to mesoscale heterogeneities such as local electric field deviations leading to random distributions of switching time constants. Building on the KAI model, the Inhomogeneous Field Mechanism (IFM) addresses the observed distribution of relaxation times by replacing the single time constant at a given field, described by equation 2, with a smooth unimodal distribution of time constants ${ }^{12,15-17}$. The IFM model has successfully been used to describe the observed polarization reversal in soft lead zirconate titanate (PZT), a lead-free Bi-based perovskite ferroelectric, and an organic ferroelectric. ${ }^{15}$

The IFM model requires that the overall switching process be a collection of component processes in which the time constants are a smooth distribution. Thus, polarization reversal involving sequential steps such as those shown schematically in Figure 1 may require an alternative description. Since multiple domain reorientation steps may not be directly discriminated in the macroscopic polarization of a sample, a more direct measurement of the component domain behaviors can improve understanding. In the present article, time-resolved high-energy x-ray diffraction is used to identify the contributing domain reorientation steps in a polycrystalline ferroelectric perovskite during application of step electric fields. The electric fields are applied in an opposite direction relative to the initial electrical poling direction. A two-step domain reorientation sequence is observed which must underpin the macroscopic strain and polarization response. Moreover, a residual stress analysis during the reversal process is used to 
discriminate the driving forces for each of the steps. A domain reversal model is proposed that accounts for these two steps and is shown to accurately describe the experimental results.

\section{$\underline{\text { Experimental }}$}

A lead zirconate titanate (PZT) polycrystalline ferroelectric (PIC151, PI Ceramic, Germany) was selected for the present investigation. This composition has been well characterized for both industrial and scientific purpose. ${ }^{18-21}$ The material is on the tetragonal side of the morphotropic phase boundary (MPB) and is chemically modified to enhance domain wall mobility. The ferroelectric coercive field, $\mathrm{E}_{\mathrm{c}}$, is approximately $1 \mathrm{kV} / \mathrm{mm}$. In situ diffraction measurements were carried out at beamline ID15A of the European Synchrotron Radiation Facility using a beam energy of $69.47 \mathrm{keV}$. Further information about the diffraction geometry can be found in Refs [22] [23] [24]. Diffracted intensities were collected using a fast-decay x-ray image intensifier, coupled with a high frame rate camera (PCO DIMAX CMOS). Samples were initially poled using a $2 \mathrm{kV} / \mathrm{mm}$ field for 5 minutes at room temperature. Step electric fields of $1.1 \mathrm{E}_{\mathrm{c}}, 1.2 \mathrm{E}_{\mathrm{c}}$ and $1.3 \mathrm{E}_{\mathrm{c}}$ were applied in a direction opposite to the initial poling field while diffraction images were collected in $1 \mathrm{~ms}$ time intervals. At these field strengths, the full polarization reversal process occurs on the milliseconds to seconds time-scale. ${ }^{15}$

The diffraction images were distortion-corrected and radially integrated into $15^{\circ}$ segments using the software package fit $2 \mathrm{~d}^{25}$ In this scattering geometry, the relative intensity of the (002) and (200) tetragonal doublet can be used to characterize the ferroelastic domain volume fractions at various angles to the applied electric field ${ }^{24,26}$ and the Bragg peak shifts can be used to investigate resultant lattice strains. The (002) and (200) tetragonal profiles were modeled using 
two Gaussian functions in order to extract relative intensities, which can then be used to calculate the volume fraction of reoriented domains following the methods of Jones et al. ${ }^{26}$ The (111) peak was modeled using a single Gaussian function to extract lattice strains.

\section{$\underline{\text { Results \& Discussion }}$}

Figure 2 shows the (002) and (200) reflections with the scattering vector, q, parallel to the applied electric field vector, E, for selected states during the reversal process. In this geometry, the relative intensities of the (002) and (200) peaks are correlated to the ferroelastic domain volume fractions aligned in the field direction, i.e. a higher intensity (002) peak relative to the (200) indicates a greater volume fraction of domains oriented with their c-axis parallel to the electric field direction. The initial un-poled sample state in Figure 2(a) is consistent with a tetragonal PZT with random domain orientations, i.e. an intensity ratio of the (002):(200) peaks of approximately 1:2. ${ }^{26}$ The initial electrically poled state, shown in Figure 2(b), exhibits a (002):(200) intensity ratio of approximately $4: 3$, indicating a significant increase in the volume fraction of domains aligned with their c-axis parallel to the electric field direction. Additionally, the poling process appears to introduce scattered intensity between the (002) and (200) tetragonal reflections. ${ }^{27}$ This additional intensity is attributed to a field-induced rhombohedral or monoclinic distortion of the parent cell which has been observed previously in this material. ${ }^{19,20}$ Figure 2(c) shows the diffraction peak profile $1 \mathrm{~ms}$ after the application of an electric field of opposite direction; at this point, the profile is similar to that observed in the initial un-poled state. Figure 2(d) shows the profile after $1000 \mathrm{~ms}$ of the applied field; the relative intensities indicate a higher volume fraction of domains aligned with their c-axes parallel to the electric field direction. The results shown in 
Figure 2 indicate a domain reorientation process that involves an intermediate ferroelastic domain state. These results are consistent with the schematic presented in Figure 1.

The time dependence of the domain reorientation process was investigated by calculating the volume fraction of switched ferroelastic domains $(\Delta \eta)$ from each measured diffraction pattern. $\Delta \eta$ scales from $-1 / 3$ to $2 / 3$, where 0 corresponds to the volume fraction of switched domains for the unpoled sample (i.e. $\eta_{002}=0$ ). ${ }^{26}$ Figure 3 (a) shows the resulting $\Delta \eta$ as a function of time after the application of step electric fields of amplitude 1.1, 1.2 and 1.3 $\mathrm{E}_{\mathrm{C}}$. For all field amplitudes, the $\Delta \eta$ during the polarization reversal process returns to a value close to that of the initial unpoled sample, i.e. 0 . This result is consistent with the qualitative observation that the intensity ratio of (002) and (200) peaks at this position returns close to the values measured in an unpoled sample (Figure 2(c) vs. 2(a)). These data reveal that the reversal mechanism occurs via sequential steps involving an intermediate ferroelastic domain state. Moreover, this observation and the results in Figure 3(a) show two distinct time constants associated with the reversal process; i) a fast component involving domain reorientation from the initial poled to an intermediate state, and ii) a slower component involving domain reorientation from the intermediate state to the state of opposite polarity.

The domain reorientation process shown in Figure 3(a) can be described using a derivative of the KAI model in which the overall domain reorientation process is described by two individual components. The components are each described by an independent KAI model. The overall model is given as,

$$
\Delta \eta(t)=\eta_{0}+2 \eta_{\rightarrow}\left(1-e^{-t / \tau_{\rightarrow}}\right)+2 \eta_{\uparrow}\left(1-e^{-t / \tau_{\uparrow}}\right)
$$


where $t$ is time elapsed, $\eta_{0}$ is the volume fraction of domains after initial poling, $\eta_{\rightarrow}$ and $\eta_{\uparrow}$ are the domain switching fractions during each sequential step, $\tau_{\rightarrow}$ and $\tau_{\uparrow}$ are the unique time constants for each step within the process (in the present work, $\tau_{\rightarrow}<<\tau_{\uparrow}$ ).

Equation 3 has been fit to the reorientation of the domains in Figure 3(a) and is shown as a solid line. All values of $\tau \rightarrow$ are less than $2 \mathrm{~ms}$; a more accurate determination of this value is not possible due to the fact that most of this step occurred faster than the time resolution of the instrumentation. Alternative techniques may need to be employed to overcome this limitation. ${ }^{35}$ The values of $\tau_{\uparrow}$ from the fits are approximately $110 \mathrm{~ms}, 16 \mathrm{~ms}$, and $3.3 \mathrm{~ms}$ for reversal field magnitudes of 1.1, 1.2 and 1.3 $\mathrm{E}_{\mathrm{C}}$, respectively. The values of $\tau_{\uparrow}$ are shown to be significantly longer than those of $\tau_{\rightarrow}$. Following the extension of the KAI model to the IFM model, it is logical to suggest that each $\tau_{\rightarrow}$ and $\tau_{\uparrow}$ may be an independent and smooth distribution of values centered on the values reported here, and could be further improved by an IFM type modeling of each of the individual steps in Equation 3.

In order to investigate the origin of the unique time constants associated with the domain reorientation process, elastic residual strains were investigated. It is known that certain grain orientations interact with domain reorientation behavior in other grains and can exhibit large elastic residual stresses. ${ }^{24,26,28,29}$ For tetragonal perovskite systems, the residual stresses can be observed by the lattice strains in (111) type diffraction peaks. Figure 4 shows the distribution of (111) lattice strains as a function of angle, $\psi$, between the diffraction vector, $\mathrm{q}$, and the applied electric field vector, $\mathrm{E}$, at selected points within the reversal process of the $1.2 \mathrm{E}_{\mathrm{C}}$ sample. In the initial poled state, a residual tensile strain of approximately $1.2 \times 10^{-3}$ is measured which is acting to compress the sample along the field direction, $\psi=0$. At the time at which the intermediate domain state is 
observed, a smaller residual strain of $-0.5 \times 10^{-3}$ exists. After the completion of the domain reorientation process, a significant tensile residual strain is again observed.

Domain wall motion can also be described using phenomenological models that involve pinning centers as a function of distance in the material. Boser ${ }^{30}$ first introduced such models and these have been developed to describe random energy landscapes of pinning potentials for use in describing effects of mobile interfaces on properties, e.g. in modeling Rayleigh behavior in dielectric and piezoelectric properties of ferroelectric materials. ${ }^{31-33}$ Tutuncu et al. added longerrange and stronger pinning potentials to this model in order to illustrate driving forces for the progressive loss of domain orientation, or deaging. ${ }^{34}$ Residual lattice strains in grain orientations that do not deform ferroelastically, as observed in the present work, is a long-range driving force for domain wall motion and can therefore also be represented using an energy landscape of pinning potentials. Specifically, the residual stress provides an extra force for the motion of domain walls. Residual tensile strains may promote the backwards motion of domain walls relative to the initial poling direction and residual compressive strains may promote the forwards motion of domain walls. A modified pinning potential landscape is shown in Figure 5 that includes a bias resulting from residual stresses of selected grain families. After poling, a metastable domain wall position is identified. Under the application of an electric field of opposite direction, the domain wall motion is driven both by the applied electric field and the elastic residual stress. The domain wall passes an equilibrium position at which the elastic residual stress is minimum. However, the driving electric field remains constant and domain wall motion proceeds in the negative direction, albeit at a slow time constant. The two significantly different time constants observed in the present work can be rationalized using such a representation and are identified in two different regions of Figure 5. 
The structural insight provided by these time-resolved diffraction measurements affords critical information for the further development of models of polarization switching. The most remarkable feature of the measured domain reorientation is that the reversal process occurs with two distinct time constants that are significantly different. This observation suggests that the models often applied to polarization reversal kinetics, the KAI and IFM models may not able to capture certain domain reorientation mechanisms wholly. This is because the KAI model assumes a single time constant for the switching process, while the IFM model assumes a smooth unimodal distribution of time constants, neither of which captures the two-step and independent time constants associated with the polarization reversal process observed here. Furthermore, because the intermediate domain state involves a local deformation of the unit cell, the two time constants should have unique signatures in the macroscopic strain. Measurement of the macroscopic strain under similar conditions and samples are shown in Figure 3(b) and confirm this. This result suggests that macroscopic strain may provide unique information about this two-step polarization reversal process in similar materials. The intermediate domain state will also have an effect on the measured macroscopic polarization and models of this behavior may be extended based on Equation 3.

\section{Conclusion}

In situ high-energy $\mathrm{x}$-ray diffraction has shown that an intermediate domain orientation state exists during polarization reversal of a tetragonal ferroelectric under application of step electric fields. The measurements uniquely identify two independent time constants reflecting a sequence of domain reorientation steps. The first and faster time constant is shown to be strongly affected by the residual stress that was developed during the initial poling of the material. The second and 
slower time constant is associated with domain wall motion acting to redevelop the elastic residual

stress. A new polarization reversal model was introduced to account for the two-step reorientation process and shown to agree quantitatively with both the measured domain volume fraction and macroscopic strain data.

JED acknowledges financial support from an AINSE Research Fellowship, ARC Discovery Project DP120103968, and travel funding provided by the International Synchrotron Access Program (ISAP) managed by the Australian Synchrotron and funded by the Australian Government. JJ and CD acknowledge the U.S. National Science Foundation award number DMR0746902. Involvement of CC was enabled from the U.S. National Science Foundation award number OISE-1129412. Experimental beamtime was provided by the European Synchrotron Radiation Facility (Exp. MA-1108).

\section{References}

${ }^{1}$ N. Bar-Chaim, M. Brunstein, J. Grunberg, A. Seidman, J. Appl. Phys. 1974, 45, 2398.

${ }^{2}$ W. Cao, Ferroelectrics. 2003, 290, 107.

${ }^{3}$ W. Zhu, L. E. Cross, Appl. Phys. Lett. 2004, 84, 2388.

${ }^{4}$ A. N. Kolmogorov, Izv. Akad. Nauk SSSR, Ser. Math, 1937, 3, 355.

${ }^{5}$ M. Avrami, J. Chem. Phys. 1940, 8, 212.

${ }^{6}$ Y. Ishibashi, H. Orihara, Integr. Ferroelectr. 1995, 9, 57.

${ }^{7}$ E. Fatuzzo, W. J. Merz, Phys. Rev. 1959, 116, 61.

${ }^{8}$ D. Pantel, Y.-H. Chu, L. W. Martin, R. Ramesh, D. Hesse, M. Alexe, J. Appl. Phys. 2010, 107, 084111.

${ }^{9}$ J. Li, Z. Liu, B. W. Wessels, J. Appl. Phys. 2010, 107, 124106.

${ }^{10}$ A. K. Tagantsev, I. Stolichnov, N. Setter, J. S. Cross, M. Tsukada, Phys. Rev. B. 2002, 66, 214109.

${ }^{11}$ A. Gruverman, B. Rodriguez, C. Dehoff, J. Waldrep, A. Kingon, R. Nemanich, J. Cross, Appl. Phys. Lett. 2005, 87, 082902.

${ }^{12}$ S. Zhukov, Y. A. Genenko, O. Hirsch, J. Glaum, T. Granzow, H. von Seggern, Phys. Rev. B. 2010, 82, 014109. 
${ }^{13}$ D. C. Lupascu, S. Fedosov, C. Verdier, J. Rodel, H. von Seggern, J. Appl. Phys. 2004, 95, 1386. ${ }^{14}$ T. M. Kamel, F. X. N. M. Kools, G. de With, J. Euro. Ceram. Soc. 2007, 27, 2471.

${ }^{15}$ Y. A. Genenko, S. Zhukov, S. V. Yampolskii, J. Schütrumpf, R. Dittmer, W. Jo, H. Kungl, M. J. Hoffmann, H. von Seggern, Adv. Funct. Mater. 2012, 22, 2058.

${ }^{16}$ O. Lohse, M. Grossmann, U. Boettger, D. Bolten, R. Waser, J. Appl. Phys. 2001, 89, 2332.

${ }^{17}$ S. Zhukov, Y. A. Genenko, H. von Seggern, J. Appl. Phys. 2010, 108, 014106.

${ }^{18}$ J. Suchanicz, D. Sitko, N. T. H. Kim-Ngan, A. G. Balogh, J. Appl. Phys. 2008, 104, 094106.

${ }^{19}$ A. B. Kounga, T. Granzow, E. Aulbach, M. Hinterstein, J. Rodel, J. Appl. Phys. 2008, 104, 024116.

${ }^{20}$ M. Hinterstein, J. Rouquette, J. Haines, P. Papet, M. Knapp, J. Glaum, H. Fuess, Phys. Rev. Lett. 2011, 107, 077602.

${ }^{21}$ M. Hinterstein, M. Hoelzel, H. Kungl, M. J. Hoffmann, H. Ehrenberg, H. Fuess, Z. Kristallogr. Krist. 2011, 226, 155.

${ }^{22}$ J. Daniels, A. Pramanick, J. Jones, IEEE T. Ultrason. Ferr. 2011, 56, 1539.

${ }^{23}$ J. Daniels, J. Appl. Crystallogr. 2008, 41, 1109.

${ }^{24}$ A. Pramanick, D. Damjanovic, J. E. Daniels, J. C. Nino, J. L. Jones, J. Am. Ceram. Soc. 2011, 94, 293.

${ }^{25}$ A. P. Hammersley, S. O. Svensson, M. Hanfland, A. N. Fitch, D. Hausermann, High Pressure Res. 1996, 14, 235.

${ }^{26}$ J. L. Jones, E. B. Slamovich, K. J. Bowman, J. Appl. Phys. 2005, 97, 034113.

${ }^{27}$ J. E. Daniels, J. L. Jones, T. R. Finlayson, J. Phys. D. Appl. Phys. 2006, 39, 5294.

${ }^{28}$ D. A. Hall, A. Steuwer, B. Cherdhirunkorn, T. Mori, P. J. Withers, J. Appl. Phys. 2004, 96, 4245.

${ }^{29}$ D. A. Hall, A. Steuwer, B. Cherdhirunkorn, P. J. Withers, T. Mori, J. Mech. Phys. Solids. 2005, $53,249$.

${ }^{30}$ O. Boser, J. Appl. Phys. 1987, 62, 1344.

${ }^{31}$ D. A. Hall, J. Mater. Sci. 2001, 36, 4575.

${ }^{32}$ D. Dragan, D. Marlyse, J. Phys. D. Appl. Phys. 1996, 29, 2057.

${ }^{33}$ P. Bintachitt, S. Jesse, D. Damjanovic, Y. Han, I. M. Reaney, S. Trolier-McKinstry, S. V. Kalinin, P. Natl. Acad. Sci. 2010, 107, 7219.

${ }^{34}$ G. Tutuncu, D. Damjanovic, J. Chen, J. L. Jones, Phys. Rev. Lett. 2012, 108, 177601.

${ }^{35}$ S. Gorfman, Crystallogr.. Rev. 2014, 20, 210. 


\section{Figure Captions}

FIG 1. Schematic representation of polarization reversal. An out of plane variant (i.e., up or down) and an in-plane variant (i.e. a right arrow) demonstrate two successive $90^{\circ}$ switching processes $\left(\tau_{\rightarrow}\right.$ or $\left.\tau_{\uparrow}\right)$ in response to step electric field.

FIG 2. (a) Initial diffraction profile of the unpoled sample, (b) Remanent state after initial poling, (c) After $1 \mathrm{~ms}$, i.e. intermediate state during polarization reversal, (d) After $1000 \mathrm{~ms}$. The solid lines represent the total fit profile, while the dashed lines are the individual peak component profiles.

FIG 3. (a) Time dependent intensity ratio of the switched domain volume fraction during polarization reversal at different field strengths with fits according to Equation (3). 
FIG 4. Distribution of (111) lattice strains as a function of angle, $\psi$, to the applied electric field vector. Tensile strains along the electric field direction act to compress the material.

FIG 5. Energy landscape of a domain wall in spatial dimension, u. (a) with no external electric field, and (b) with an external electric field opposite to the initial bias direction. The time constants are highlighted in (b), where switching at rate $\tau_{\rightarrow}$ cannot commence until switching at rate $\tau_{\uparrow}$ has completed. Dashed lines indicate the energy offset due to residual stresses (a), and residual stresses and electric field (b).

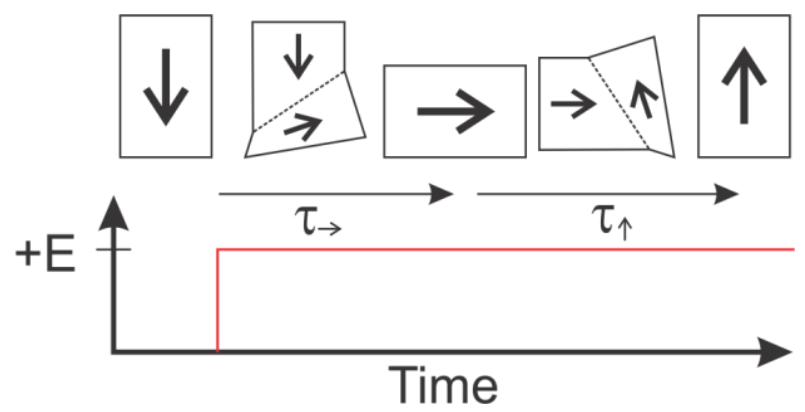

FIG 1. 


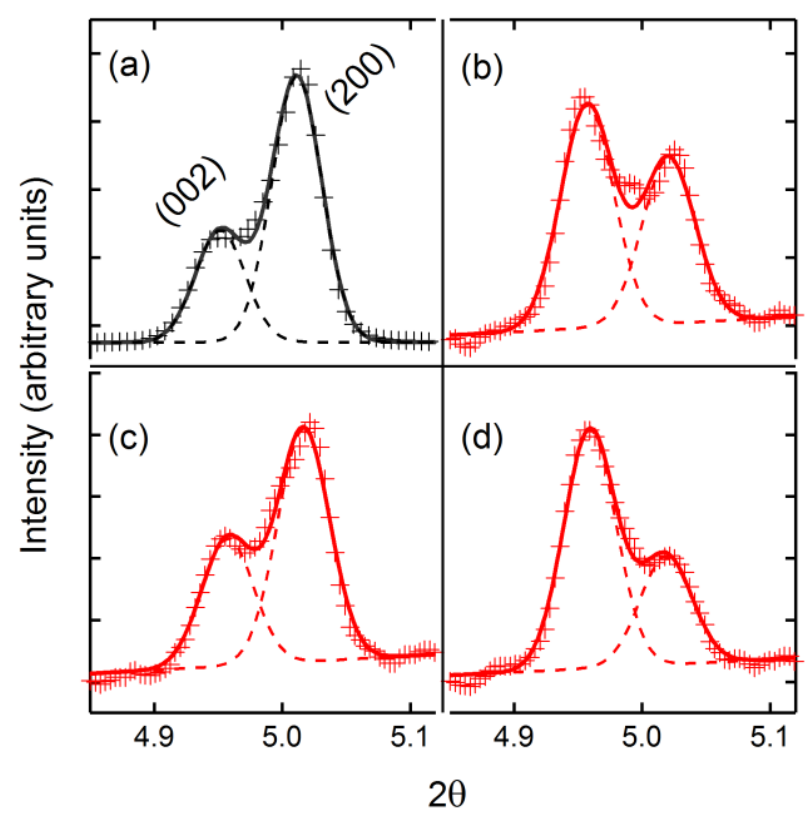

FIG 2. 


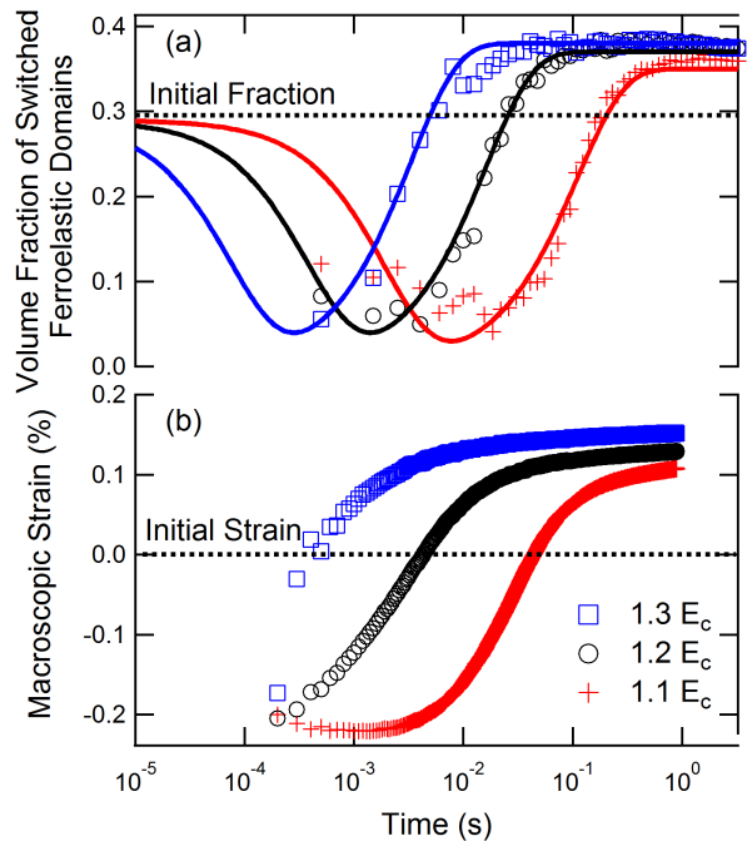

FIG 3. 


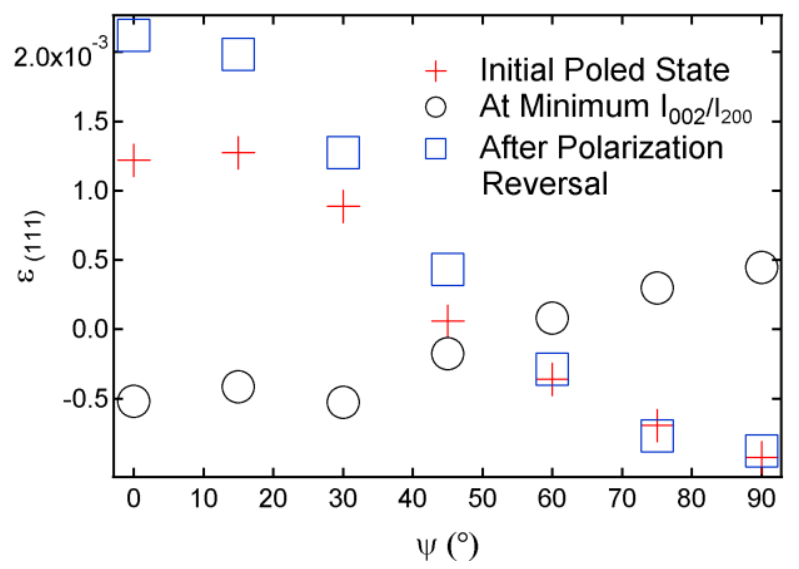

FIG 4. 


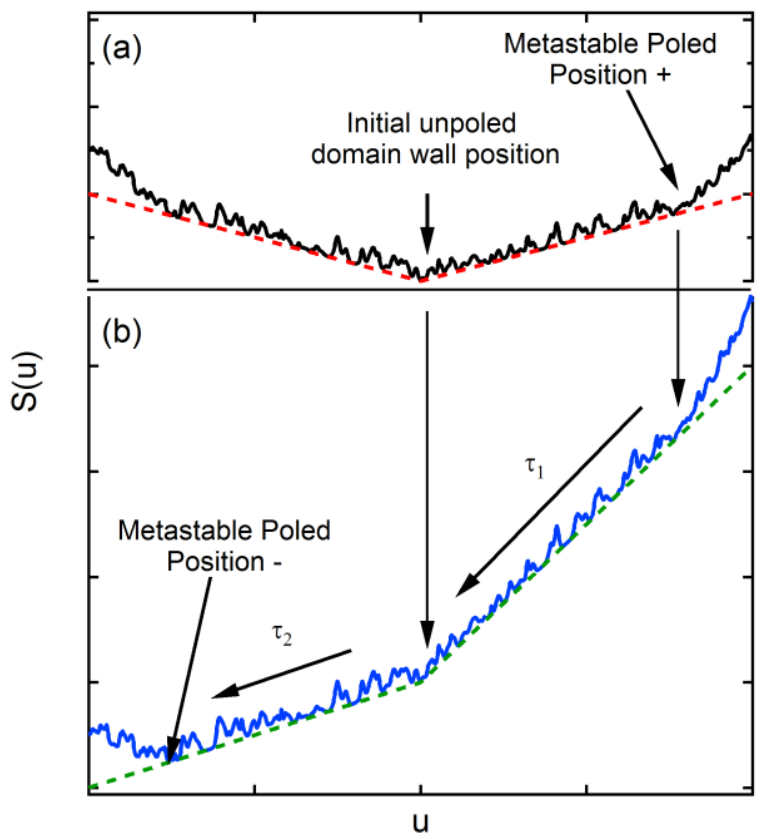

FIG 5. 